\title{
Clinical differences among the elderly admitted to the emergency department for accidental or unexplained falls and syncope
}

\author{
Giuseppe Pasqualetti' \\ Valeria Calsolaro' \\ Giacomo Bini' \\ Umberto Dell'Agnello' \\ Marco Tuccori \\ Alessandra Marino ${ }^{2}$ \\ Alice Capogrosso-Sansone ${ }^{2}$ \\ Martina Rafanelli ${ }^{3}$ \\ Massimo Santini ${ }^{4}$ \\ Eugenio Orsitto ${ }^{4}$ \\ Andrea Ungar ${ }^{3}$ \\ Corrado Blandizzi ${ }^{2}$ \\ Fabio Monzani' \\ On behalf of the \\ ANCESTRAL-ED study \\ group \\ 'Geriatrics Unit, ${ }^{2}$ Pharmacology \\ Unit, Department of Clinical and \\ Experimental Medicine, University of \\ Pisa, Pisa, ${ }^{3}$ Syncope Unit, Geriatric and \\ Intensive Care Medicine, AOU Careggi \\ and University of Florence, Florence, \\ ${ }^{4}$ Emergency Department, University \\ Hospital of Pisa, Pisa, Italy
}

This article was published in the following Dove Press journal:

Clinical Interventions in Aging

13 April 2017

Number of times this article has been viewed

\begin{abstract}
It is difficult to distinguish unexplained falls (UFs) from accidental falls (AFs) or syncope in older people. This study was designed to compare patients referred to the emergency department (ED) for AFs, UFs or syncope. Data from a longitudinal study on adverse drug events diagnosed at the ED (ANCESTRAL-ED) in older people were analyzed in order to select cases of AF, syncope, or UF. A total of 724 patients (median age: 81.0 [65-105] years, $66.3 \%$ female) were consecutively admitted to the ED (403 AF, 210 syncope, and 111 UF). The number of psychotropic drugs was the only significant difference in patients with AF versus those with UF (odds ratio [OR] 1.44; 95\% confidence interval 1.17-1.77). When comparing AF with syncope, female gender, musculoskeletal diseases, dementia, and systolic blood pressure $>110 \mathrm{mmHg}$ emerged as significantly associated with AF (OR 0.40 [0.27-0.58], 0.40 [0.24-0.68], 0.35 [0.14-0.82], and 0.31 [0.20-0.49], respectively), while valvulopathy and the number of antihypertensive drugs were significantly related to syncope (OR 2.51 [1.07-5.90] and 1.24 [1.07-1.44], respectively). Upon comparison of UF and syncope, the number of central nervous system drugs, female gender, musculoskeletal diseases, and SBP $>110 \mathrm{mmHg}$ were associated with UF (OR 0.65 [0.50-0.84], 0.52 [0.30-0.89], 0.40 [0.20-0.77], and 0.26 [0.13-0.55]), respectively. These results indicate specific differences, in terms of demographics, medical/pharmacological history, and vital signs, among older patients admitted to the $\mathrm{ED}$ for $\mathrm{AF}$ and syncope. UF was associated with higher use of psychotropic drugs than AF. Our findings could be helpful in supporting a proper diagnostic process when evaluating older patients after a fall.
\end{abstract}

Keywords: older people, frailty, fall, accidental fall, syncope

\section{Introduction}

Falls represent a significant proportion of visits to the emergency department (ED) in the elderly. ${ }^{1,2}$ Some naturalistic studies showed that $~ 15 \%$ of ED admissions in older people are related to falls. ${ }^{1,3,4}$ The annual incidence of falls in community-dwelling subjects older than 65 years is $\sim 28 \%-35 \%$, up to $40 \%$ in those older than 75 years. ${ }^{5}$ Forty percent of falls are due to accidental causes, while a substantial percentage remains unexplained, ${ }^{6}$ suggesting a possible overlap between the pathophysiology of falls and syncope. ${ }^{7,8}$ Interestingly, $20 \%$ of patients with syncope have amnesia for the loss of consciousness, ${ }^{9}$ and nearly two-thirds of older adults with orthostatic hypotension, who experience a fall, deny a loss of consciousness. ${ }^{10}$ Both accidental fall (AF) and syncope may cause traumatic injuries and are associated with poor global short- and long-term outcomes. ${ }^{11-13}$ Moreover, the economic burden of injuries due to AF or syncope represents one of the greatest sources of expenditure for traumatic patients..$^{14,15}$ 
In older people, AFs are generally related to an impaired physical performance and functional reserves and, in general, represent an epiphenomenon of the "frailty syndrome". ${ }^{11}$ By definition, syncope is a transient loss of consciousness caused by a transient reduction of global cerebral blood flow, whose diagnosis is mostly determined by a careful clinical history collection. ${ }^{16}$ A clear detection of the exact dynamic of the fall is quite challenging, especially in patients with moderate to severe amnestic cognitive impairment and several comorbidities (neurological, cardiac, and so on), ${ }^{14,17}$ and in many cases, the underlying mechanism remains unexplained. Indeed, up to $30 \%$ of older people with unwitnessed syncope report AF rather than syncope. ${ }^{18}$ Moreover, in some cases, the pathophysiology of syncope (reduction of cerebral blood flow, eg, orthostatic hypotension) does not lead to a complete loss of consciousness but produces only transient-related symptoms, such as dizziness, vertigo, palpitation, and weakness, that may favor a fall. ${ }^{14,15}$ Likewise, similar prodromes may be due to vestibular disorders or psychotropic drug adverse effects. ${ }^{19}$ In order to provide proper therapeutic interventions, it is crucial to recognize the pathophysiology of falls, especially at the first-line evaluation in the ED, where a misdiagnosis can adversely affect the long-term outcome. ${ }^{7,20}$ Given the difficulty of clearly distinguishing whether an unexplained fall (UF) in older people results from a syncope or accidental causes, we designed a clinical study to compare older patients (aged $>65$ years) consecutively admitted to the ED of the University Hospital of Pisa for syncope, AF, or UF. The patients were allocated to the AF group according to Tinetti et $\mathrm{a}^{21}$ criteria, and in order to clearly distinguish those patients from those who experienced an UF, we added the following criterion to this group: the absence of any prodrome (feeling of faint, palpitation, vertigo, and dizziness) before falling. Patients who experienced falls without a complete loss of consciousness but with prodrome symptoms and/or amnesia for the event were allocated to the UF group. Patients were included in the syncope group according to the European Society of Cardiology Guideline on syncope. ${ }^{16}$

\section{Patients and methods}

This study is part of a larger prospective study on adverse drug reactions diagnosed at the $\mathrm{ED}$ of the University Hospital of Pisa (ANCESTRAL-ED). ${ }^{22}$ In detail, older patients (aged $>65$ years) referred to the ED were consecutively enrolled at the triage area by a team comprising of geriatricians and pharmacologists. Before any further study procedure, each enrolled patient gave written informed consent to partake in the study. In cases when patients were unable to provide consent, we took the informed consent from the legally authorized delegate. Each patient with mild to moderate medical problems (red code was excluded for impossibility in collecting the data due to the severity of cases) were interviewed. In the case of inability to properly answer questions, the informant was interviewed and the answers recorded in a database. A detailed medical (past and recent) and pharmacological history, along with vital signs and laboratory data (if available), were collected. An online database (Microme$\operatorname{dex}^{\circledR}$ ) was consulted to evaluate the expected drug reactions and possible drug-drug interactions. All the data were entered into a proper database. The Ethical Committee of the University Hospital of Pisa approved the study protocol, which was designed in full accordance with the Declaration of Helsinki.

The enrolled patients were allocated to the AF group according to Tinetti et al: ${ }^{22}$

$[\ldots]$ an event which results in a person coming to rest unintentionally on the ground or other lower level, not as the result of a major intrinsic event (such as stroke) or overwhelming hazard.

The absence of any prodrome (feeling of faint, palpitation, vertigo, and dizziness) before falling was added as further inclusion criterion. Patients were included in the syncope group according to the European Society of Cardiology Guideline on syncope: ${ }^{16}$

Syncope is a T-LOC due to transient global cerebral hypoperfusion characterized by rapid onset, short duration, and spontaneous complete recovery.

Finally, patients who experienced falls without a complete loss of consciousness but with prodrome and/or amnesia for the event were allocated to the UF group. We excluded patients with presyncope symptoms without a fall or cases of uncertain dynamic (eg, patients with amnesia or unable to refer the dynamic of fall, without witnesses). Demographic data, past medical history, pharmacological information (number and class of drugs), laboratory data, and vital signs (heart rate, systolic blood pressure [SBP], diastolic blood pressure [DBP], peripheral oxygen saturation, and body temperature) were extracted from the ANCESTRAL-ED database. For each patient, we also took into consideration the presence of major chronic diseases, grouped in general classes (systemic hypertension, neurological disorders [chronic vascular encephalopathy, Parkinson's disease, and other neurodegenerative disorders excluding dementia], any kind of dementia, cardiovascular diseases, musculoskeletal diseases [osteoporosis/sarcopenia with or without previous 
fractures and osteoarthritis], anemia, and diabetes mellitus). The Cumulative Illness Rating Scale Severity (CIRS-S) and Cumulative Illness Rating Scale Comorbidity (CIRS-C) ${ }^{23}$ were estimated for each patient.

\section{Statistical analysis}

Statistical analysis was performed using SPSS Version 21 (IBM Corporation, Armonk, NY, USA). Normally distributed variables were described as mean and standard deviation (SD) and were compared across groups by independent $t$-test. Nonnormally distributed variables were described as median and range and compared using the Mann-Whitney $U$-test, while categorical variables were compared using the $\chi^{2}$ test. The Bonferroni correction was applied for multiple comparisons. Multiple logistic regression analysis (backward; probability for stepwise: 0.05 entry, 0.05 removal) with each clinically and statistically significant variable as covariates and the presence of AF/syncope, $\mathrm{AF} / \mathrm{UF}$, or syncope/UF as dependent variables was performed. Age, the number of daily administered drugs, the number of daily antihypertensive drugs, and central nervous system (CNS) medications were considered as ordinal variables. For multivariate regression, missing values were imputed as values randomly extracted from the range of each specific parameter by Excel function (Microsoft ${ }^{\circ}$, Office). Bootstrapping analysis with 1,000 samples was also performed. Statistical significance was assigned for $P<0.05$.

\section{Results}

We enrolled 724 patients (458 females, median age 81.0 [range 65-105] years), 19.5\% (95\% confidence interval [CI] 18.2-20.8) of the whole cohort of the ANCESTRAL-ED study: 403 patients with AF, 210 patients with syncope, and 111 patients with UF. Patients' characteristics are reported in Table 1. Patients with syncope were significantly younger, with a higher prevalence of males than those with AF ( $P<0.05$ for both). The prevalence of musculoskeletal diseases was significantly higher in the AF and UF groups than in the syncope group ( $P<0.05$ for both).

Data on SBP and DBP were available for 587 patients (300 patients with AF, 191 patients with syncope, and 96 patients with UF). The mean values of SBP and DBP (mmHg) were significantly lower in the syncope group with respect to both AF and UF groups ( $P<0.05$ for both). The median number of daily administered drugs was significantly higher in UF patients than in AF patients ( 5 vs 4 daily, $P<0.05$ ), while those with syncope took more antihypertensive drugs than those with AF $(P<0.05)$. The daily use of CNS drugs was significantly greater in the UF group than either in the
AF group or in the syncope group ( $P<0.05$ for both). The principal pharmacological classes of daily administered drugs are reported in Table 2. AF and syncope patients significantly differed for the use of antihypertensive medications, particularly for angiotensin-converting enzyme inhibitors and angiotensin II receptor blockers (ACEIs-ARBs), alpha blockers, and diuretics ( $P<0.05$ for all). The use of nitrates was higher in the syncope group than in both the AF and UF groups $(P<0.05)$. The use of at least one psychotropic drug was significantly more frequent in patients with UF than in those with syncope $(P<0.05)$. A higher prevalence of UF patients was taking sedative/hypnotic or antiepileptic/ GABAergic drugs with respect to either AF patients or syncope patients $(P<0.05)$.

\section{Multiregression analysis}

The clinical parameters reported in Table 1 were analyzed by univariate regression analysis, comparing AFs vs UFs, AFs vs syncope, and syncope vs UFs. The results are reported in Table 1.

Multivariate regression analyses were performed selecting as independent variables those resulted significant in each univariate analysis of the above described comparisons. In order to simplify the comparison between groups, we transformed SBP values in a dichotomous variable by choosing the upper value of the first tertile as cutoff. By examining AF and syncope as dependent variables, the female gender, musculoskeletal diseases, dementia, and SBP $>110 \mathrm{mmHg}$ emerged as significantly associated with AF, while valvulopathy and the number of daily administered antihypertensive drugs were significantly related to syncope. By comparing UF and syncope, the number of daily administered CNS drugs, female gender, musculoskeletal diseases, and $\mathrm{SBP}>110 \mathrm{mmHg}$ resulted as significantly associated with UFs. Finally, by comparing AFs and UFs, the number of CNS drugs emerged as the only significant parameter significantly associated with UFs (Table 3 ). As a sensitivity test, the bootstrapping method confirmed all the significances of the multiregression analysis.

\section{Discussion}

This study confirms that, in older adults, falls and syncope are striking health problems that account for approximately one-sixth of ED admissions of older people. ${ }^{1,2}$ Moreover, an accurate medical and pharmacological history along with SBP measurement, directly collected at the triage area, emerged as useful tools to identify the dynamic of the fall. Indeed, the presence of cardiac valvulopathy and the use of 


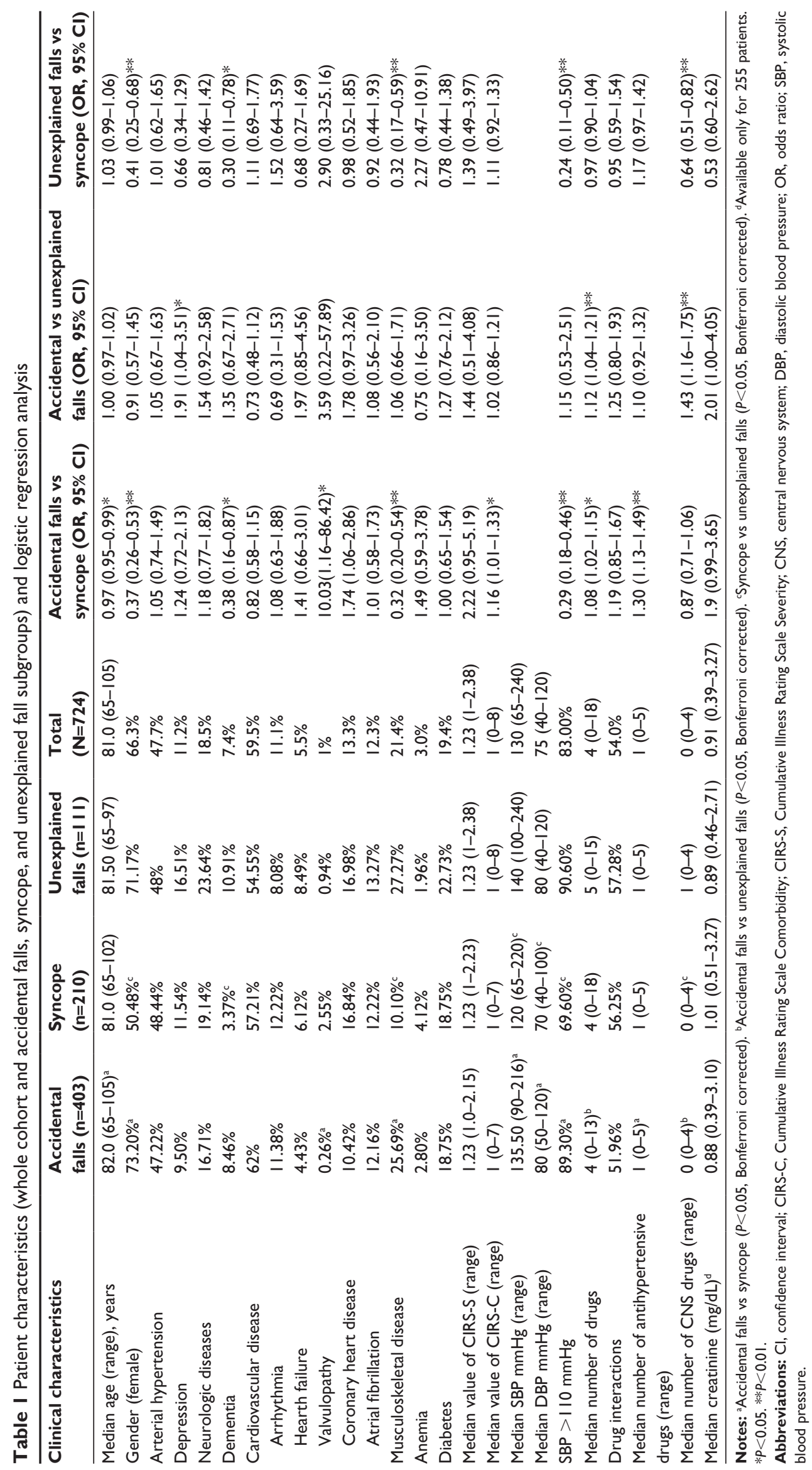


Table 2 Daily administered drugs in the three subgroups (accidental falls, syncope, and unexplained falls)

\begin{tabular}{|c|c|c|c|c|}
\hline Drug type & $\begin{array}{l}\text { Accidental falls } \\
(n=403)(\%)\end{array}$ & $\begin{array}{l}\text { Syncope } \\
(n=2 \mid 0) \text { (\%) }\end{array}$ & $\begin{array}{l}\text { Unexplained falls } \\
(n=I I I)(\%)\end{array}$ & $\begin{array}{l}\text { Total } \\
(\mathrm{N}=724)(\%)\end{array}$ \\
\hline $\mathrm{AH}$ drugs & $59.2^{\mathrm{a}}$ & 70.3 & 64.2 & 63.2 \\
\hline ACEI-ARBs & $34.8^{\mathrm{a}}$ & 44 & 35.8 & 37.6 \\
\hline Ca-blocker DHP & 11.9 & 11.5 & 15.6 & 12.4 \\
\hline Ca-blocker NDHP & 5.0 & 2.9 & 5.5 & 4.4 \\
\hline Beta-blockers & $\mathrm{I}, 742$ & 24.9 & 16.5 & 19.4 \\
\hline Alpha blocker & $6.2^{\mathrm{a}}$ & 13.4 & 10.1 & 8.9 \\
\hline Diuretics & $25.4^{\mathrm{a}}$ & 36.8 & 30.3 & 29.4 \\
\hline Nitrates & $7.2^{\mathrm{a}}$ & $13.4^{\mathrm{c}}$ & 3.7 & 8.5 \\
\hline Antiarrhythmics & 6.5 & 10.5 & 11.0 & 8.3 \\
\hline Digoxine & 5.2 & 4.8 & 6.4 & 5.3 \\
\hline Hypoglycemic agents & 15.2 & 17.2 & 16.4 & 16.0 \\
\hline Psychotropic drugs & 38.3 & $33.5^{\mathrm{c}}$ & 50.0 & 38.4 \\
\hline Antihistamines & 2.2 & 1.0 & 3.7 & 2.1 \\
\hline Anticholinergic & 1.0 & 2.9 & 2.8 & 1.8 \\
\hline Antidepressive & 21.4 & 14.4 & 24.8 & 19.9 \\
\hline Sedative-hypnotic drugs & $14.9^{\mathrm{b}}$ & $12^{c}$ & 29.4 & 16.3 \\
\hline Antipsychotic & 5.2 & 5.3 & 8.3 & 5.7 \\
\hline Antiepilectic/GABAergic & $7.5^{\mathrm{b}}$ & $7.2^{\mathrm{c}}$ & 18.3 & 9.0 \\
\hline $\mathrm{AChEi}$ & 1.5 & 0.5 & 1.8 & 1.3 \\
\hline
\end{tabular}

Notes: accidental falls vs syncope $\left(P<0.05\right.$, Bonferroni corrected). ${ }^{b}$ Accidental falls vs unexplained falls $(P<0.05$, Bonferroni corrected). 'Syncope vs unexplained falls $(P<0.05$, Bonferroni corrected).

Abbreviations: ACEI-ARBs, angiotensin-converting enzyme inhibitor and angiotensin II receptor blockers; AChEi, acetylcholinesterase inhibitor; AH, antihypertensive; BDZ, benzodiazepine; DHP, dihydropyridine; NDHP, nondihydropyridine.

antihypertensive medications resulted as potential risk factors for syncope, while female gender and SBP $>110 \mathrm{mmHg}$, along with the presence of musculoskeletal diseases or dementia, characterized patients with AFs. These latter parameters were also associated with UFs compared to syncope, while only a higher number of daily drugs, especially psychotropic, distinguished UFs from AFs.

UFs are variably defined in the literature, but none of the definitions clearly distinguishes between falls related to intrinsic causes affecting the postural tone, such as transient cerebral hypoperfusion or balance alterations, and those just caused by reduced physical performance, as observed in frail patients. ${ }^{24,25}$ In the current study, we have included all the patients who experienced amnesia and/or any prodrome before falling down in the UF group. Despite these premises, our results suggest that UFs and AFs may share a common pathophysiological process except for the use of drugs active on the CNS, favoring balance alteration.

In detail, we found a significant association between age and AFs as compared to syncope but not in case of UFs. It is well recognized that aging may act as a risk factor for both falls and syncope; ${ }^{16,26}$ however, it should be underlined that the overall patient cohort included a high number of oldest old people, characterized by frailty that leads to physical impairment. A higher prevalence of females among AFs and UFs as compared to an equivalent gender distribution in patients with syncope was also observed. The higher risk of falling, extensively reported in females, is generally ascribed to the higher prevalence of musculoskeletal diseases. ${ }^{27,28,44}$ However, our data only partially support this hypothesis since, by multiregression analysis, both female gender and musculoskeletal diseases emerged as independent predictors of either AFs or UF as compared to syncope. Accordingly, no relationship has been reported between gender and syncope (including any causes) in older people. ${ }^{16}$

The history of dementia was significantly associated with both AFs and UFs in comparison to syncope. This finding is in line with a previous report that documented a greater risk of falling for patients with dementia. ${ }^{29}$ Nonetheless, given that dementia was three times more prevalent in patients with UF than in those with syncope, it should not be excluded that a proportion of patients with dementia could have suffered from a syncope instead of an UF because of memory problems. However, if we look at the characteristics of each study group, patients with UF resulted more similar to those with AF than those with syncope. Moreover, dementia per se could act as a risk factor for falls as previously reported..$^{24,29,42}$ Thus, the above described potential bias may only account for a small percentage of cases and marginally affect the results.

Another important clinical parameter strongly associated with both AFs and UFs in comparison to syncope was systemic blood pressure, either systolic or diastolic. Accordingly, $\sim 90 \%$ of patients with AFs and UFs showed 


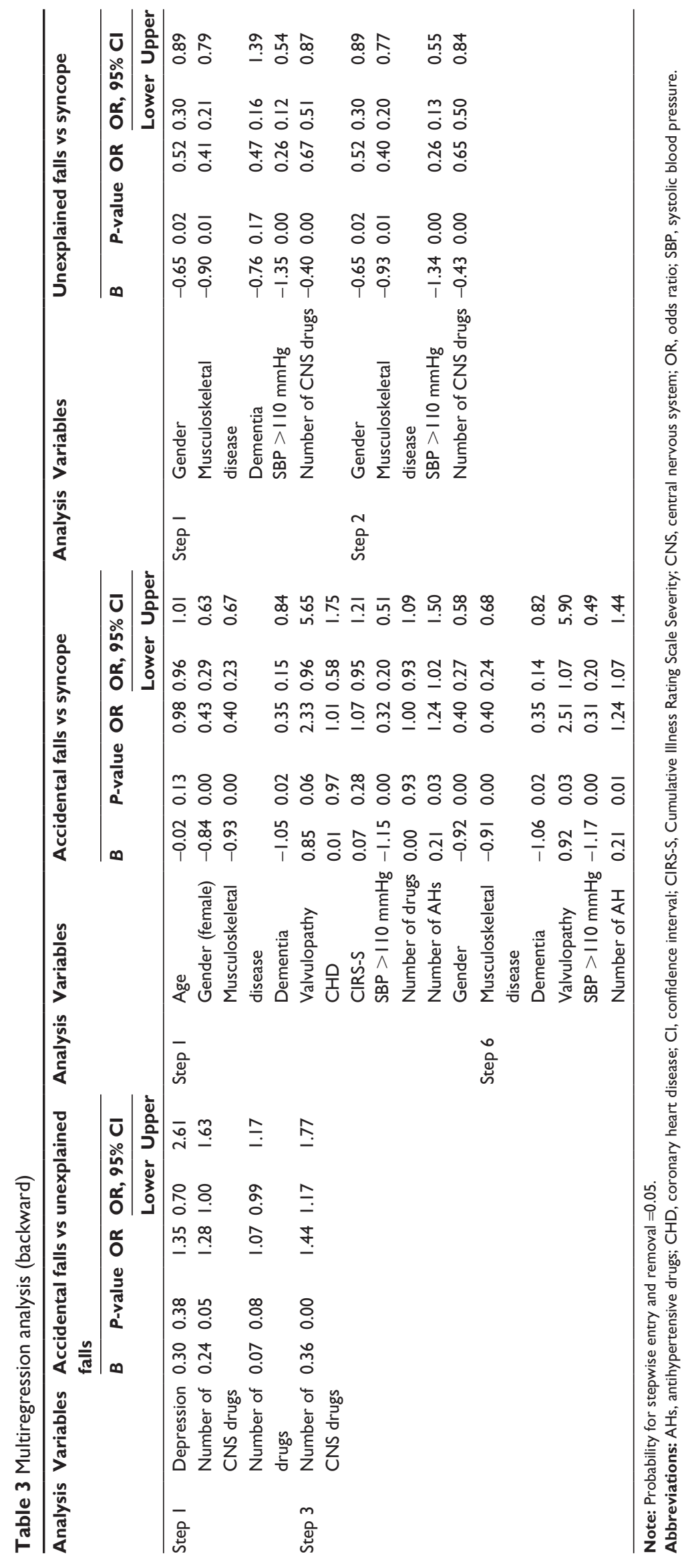


SBP values $>110 \mathrm{mmHg}$ and one-third of those with syncope showed SBP values $<110 \mathrm{mmHg}$. This finding is consistent with previous reports showing an excess use of antihypertensive drugs in older patients with syncope..$^{30,31,43}$ However, we found that the number of daily drugs was associated with syncope and UFs with respect to AFs. Anyway, this parameter was removed from the multivariate analysis in all our comparisons, suggesting the presence of possible confounders such as the use of specific drug classes (psychotropics and antihypertensives). Indeed, we observed a significant association between syncope and alpha blockers, diuretics, or nitrates, while CNS drugs, especially sedative-hypnotic medications, were mainly associated with UFs. Interestingly, the number of CNS drugs was the most important factor that distinguishes AFs from UFs. These findings may suggest that most of the UFs are AFs caused by the detrimental effects of psychotropic drugs. The association between such drugs and falls or fall-related injuries has been extensively investigated, and several pharmacological mechanisms have been proposed. ${ }^{32}$ Of interest, van der Velde et $\mathrm{al}^{33}$ observed that the withdrawal of CNS drugs in patients at risk of fall may reduce the incidence of new events.

At multivariate analysis, the history of cardiac valvular disease was significantly associated with syncope when compared with AFs. Accordingly, the presence of valvulopathy, particularly aortic stenosis, is a well-documented risk factor for syncope. ${ }^{34}$ The diagnosis of valvulopathy was done at the ED on the basis of the medical history; this circumstance may explain the relative small prevalence of valvulopathy with respect to that expected for the general population in western countries. ${ }^{34,35}$

Overall, our data suggest that an actual difference in terms of burden of comorbidity does not exist among elderly patients with different types of falls. This observation could be explained by a high proportion of very old people within each group of patients. Indeed, syncope is frequently associated with elderly comorbidities, such as heart disease (heart failure, structural heart diseases, and so on) and cerebrovascular and autonomic diseases (baroreceptor and vasovagal reflex alterations), ${ }^{16}$ while AFs usually result from decrements of muscle strength, vision, and vestibular reflex, which all are associated with the aging process $^{36}$ and the so-called frailty syndrome. ${ }^{37,45}$ Since all the above mentioned comorbidities, usually associated with syncope, play a role also in the determinism of the frailty syndrome, ${ }^{38,39}$ frailty and clinical complexity may represent common phenotypes and could account for the overlap between syncope and falls. ${ }^{40}$
The large number of consecutively enrolled patients and the prospective design mainly represent the strength of this study. Nonetheless, some limitations should be properly taken into account while interpreting the results. We performed a naturalistic study examining only an ED population. Several missing values were present for laboratory data, and the analyses were cross-sectional without further analyses during follow-up. Our classification of pharmacological categories could differ from previous reports. We did not investigate the causes of syncope and did not perform a direct analysis of frailty (eg, gait speed and muscle strength).

\section{Conclusion}

Overall, the current data confirm the presence of perceptible clinical differences among older people who refer to ED for syncope or AF. Patients with UFs, frequently preceded by prodrome, show similar demographic and medical history as compared to those with AFs but take more psychotropic medications. These clinical differences should be taken into account for a proper diagnostic process and correct classification as well as to possibly reduce the relapse rate. Future large, longitudinal studies aimed at evaluating the actual helpfulness of such factors in discriminating the cause of UF are warranted.

\section{Acknowledgments}

We thank the ANCESTRAL-ED study investigators, Sara Bernardini (Geriatrics Unit, Department of Clinical and Experimental Medicine, University of Pisa, Italy), Paola De Feo (Geriatrics Unit, Department of Clinical and Experimental Medicine, University of Pisa), Maria Giovanna Malatesta (Geriatrics Unit, Department of Clinical and Experimental Medicine, University of Pisa), Sabrina Montagnani (Pharmacology Unit, Department of Clinical and Experimental Medicine, University of Pisa, Italy), Amhed Amedeo Qasem (Geriatrics Unit, Department of Clinical and Experimental Medicine, University of Pisa), Roberta Sieli (Geriatrics Unit, Department of Clinical and Experimental Medicine, University of Pisa), Elisa Ruggiero (Pharmacology Unit, Department of Clinical and Experimental Medicine, University of Pisa), Alessandra Saporiti (Pharmacology Unit, Department of Clinical and Experimental Medicine, University of Pisa), Alessandro Cipriano (Emergency Department, University Hospital of Pisa, Italy), Irma Convertino (Pharmacology Unit, Department of Clinical and Experimental Medicine, University of Pisa), Sara Felici (Pharmacology Unit, Department of Clinical and Experimental Medicine, University of Pisa), Stefania Mantarro (Pharmacology Unit, Department of 
Clinical and Experimental Medicine, University of Pisa), Maria Teresa Galiulo (Pharmacology Unit, Department of Clinical and Experimental Medicine, University of Pisa), and Alice Ceccofiglio (Syncope Unit, Geriatric and Intensive Care Medicine, AOU Careggi and University of Florence, Italy), and all the ED employees of the University Hospital of Pisa for their kind cooperation. The study was funded by the Italian Ministry of Health (young researcher: GR-201102351231) and the Tuscan Region Authority with a grant for drug safety active surveillance programs issued by the Italian Drug Agency (Agenzia Italiana del Farmaco).

\section{Author contributions}

FM was the coordinator of the study and took part together with GP in the study design and study conduction along with data analysis and interpretation. They also participated in article writing. VC, GB, UDA, MT, AM, and AC-S took part in patient enrollment, study conduction, and data entry. $\mathrm{VC}$, GB, and UDA also participated in data analysis and article writing. MR and AU contributed to data interpretation and article revision. $\mathrm{MS}, \mathrm{EO}$, and $\mathrm{CB}$ were investigators of the ANCESTRAL-ED study and participated in article writing and revision. All authors contributed toward data analysis, drafting and critically revising the paper and agree to be accountable for all aspects of the work. All the researchers of the ANCESTRAL-ED study group took part in the study conduction at the ED.

\section{Disclosure}

The authors report no conflicts of interest in this work.

\section{References}

1. Stalenhoef PA, Crebolder HF, Knottnerus JA, Van der Horst F. Incidence, risk factors, and consequences of falls among older people subjects living in the community: a criteria-based analysis. Eur J Public Health. 1997;7(3):328-334.

2. Carpenter CR, Avidan MS, Wildes T, Stark S, Fowler SA, Lo AX. Predicting geriatric falls following an episode of emergency department care: a systematic review. Acad Emerg Med. 2014;21(10):1069-1082.

3. Sun BC, Emond JA, Camargo CA Jr. Characteristics and admission patterns of patients presenting with syncope to U.S. emergency departments, 1992-2000. Acad Emerg Med. 2004;11(10):1029-1034.

4. Sun BC, Emond JA, Camargo CA Jr. Direct medical costs of syncope related hospitalizations in the United States. Am J Cardiol. 2005;95(5): 668-671.

5. Rubenstein LZ. Falls in older people: epidemiology, risk factors and strategies for prevention. Age Ageing. 2006;35(suppl 2):ii37-ii41.

6. Richardson DA, Bexton RS, Shaw FE, Kenny RA. Prevalence of cardioinhibitory carotid sinus hypersensitivity in patients 50 years or over presenting to the accident and emergency department with "unexplained" or "recurrent" falls. Pacing Clin Electrophysiol. 1997;20(3 pt 2):820-823.

7. Tan MP, Kenny RA. Cardiovascular assessment of falls in older people. Clin Interv Aging. 2006;1(1):57-66.
8. Dey AB, Bexton RS, Tyman MM, Charles RG, Kenny RA. The impact of a dedicated "syncope and falls" clinic on pacing practice in northeastern England. Pacing Clin Electrophysiol. 1997;20(3 pt 2): 815-817.

9. McIntosh SJ, Lawson J, Kenny RA. Clinical characteristics of vasodepressor, cardioinhibitory, and mixed carotid sinus syndrome in the elderly. Am J Med. 1993;95(2):203-208.

10. Ward C, Kenny RA. Reproducibility of orthostatic hypotension in symptomatic elderly. Am J Med. 1996;100(4):418-422.

11. Tinetti ME. Preventing falls in older people persons. $N$ Engl J Med. 2003;348:41-49.

12. Tinetti ME, Williams CS. Falls, injuries due to falls, and the risk of admission to a nursing home. N Engl J Med. 1997;337(18):1279-1284.

13. Marottoli RA, Berkman LF, Cooney LM. Decline in physical function following hip fracture. J Am Geriatr Soc. 1992;40(2):861-866.

14. Ungar A, Rafanelli M, Iacomelli I, et al. Fall prevention in the older people. Clin Cases Miner Bone Metab. 2013;10(2):91-95.

15. Rubenstein LZ, Josephson KR. The epidemiology of falls and syncope. Clin Geriatr Med. 2002;18(2):141-158.

16. Moya A, Sutton R, Ammirati F, et al. Task Force for the Diagnosis and Management of Syncope; European Society of Cardiology (ESC); European Heart Rhythm Association (EHRA); Heart Failure Association (HFA); Heart Rhythm Society (HRS), Guidelines for the diagnosis and management of syncope (version 2009). Eur Heart $J$. 2009;30(2):2631-2637.

17. Parry SW, Steen IN, Baptist M, Kenny RA. Amnesia for loss of consciousness in carotid sinus syndrome: implications for presentation with falls. J Am Coll Cardiol. 2005;45(11):1840-1843.

18. Shaw FE. Falls in cognitive impairment and dementia. Clin Geriatr Med. 2002;18(2):159-173.

19. Milos V, Bondesson A, Magnusson M, Jakobsson U, Westerlund T, Midlöv P. Fall risk-increasing drugs and falls: a cross-sectional study among older people patients in primary care. BMC Geriatr. 2014; $14: 40$.

20. Soteriades ES, Evans JC, Larson MG, et al. Incidence and prognosis of syncope. N Engl J Med. 2002;347(12):878-885.

21. Tinetti ME, Speechley M, Ginter SF. Risk factors for falls among older people persons living in the community. $N$ Engl J Med. 1988; 319(26): 1701-1707.

22. Marino A, Capogrosso-Sansone A, Tuccori M, et al. Expected and actual adverse drug-drug interactions in elderly patients accessing the emergency department: data from the ANCESTRAL-ED study. Expert Opin Drug Saf. 2016;15(sup2):45-50.

23. Parmalee PA, Thuras PD, Katz IR, Lawton MP. Validation of the Cumulative Illness Rating Scale in a geriatric residential population. J Am Geriatr Soc. 1995;43(2):130-137.

24. Masud T, Morris RO. Epidemiology of falls. Age Ageing. 2001; 30(suppl 4):3-7.

25. Shaw FE, Kenny RA. The overlap between syncope and falls in the older people. Postgrad Med J. 1997;73(864):635-639.

26. Landi F, Liperoti R, Russo A, et al. Sarcopenia as a risk factor for falls in elderly individuals: results from the il SIRENTE study. Clin Nutr. 2012;31(5):652-658.

27. Ambrose AF, Paul G, Hausdorff JM. Risk factors for falls among older adults: a review of the literature. Maturitas. 2013;75(1):51-61.

28. Duckham RL, Procter-Gray E, Hannan MT, Leveille SG, Lipsitz LA, $\mathrm{Li}$ W. Sex differences in circumstances and consequences of outdoor and indoor falls in older adults in the MOBILIZE Boston cohort study. BMC Geriatr. 2013;13:133.

29. Ungar A, Mussi C, Ceccofiglio A, et al. Etiology of syncope and unexplained falls in elderly adults with dementia: syncope and dementia (SYD) study. J Am Geriatr Soc. 2016;64(8):1567-1573.

30. Rafanelli M, Ruffolo E, Chisciotti VM, et al. Clinical aspects and diagnostic relevance of neuroautonomic evaluation in patients with unexplained falls. Aging Clin Exp Res. 2014;26(1):33-37. 
31. Mussi C, Ungar A, Salvioli G, et al. Evaluation of Guidelines in Syncope Study 2 Group. Orthostatic hypotension as cause of syncope in patients older than 65 years admitted to emergency departments for transient loss of consciousness. J Gerontol A Biol Sci Med Sci. 2009;64(7):801-806.

32. Huang AR, Mallet L, Rochefort CM, Eguale T, Buckeridge DL, Tamblyn R. Medication-related falls in the elderly: causative factors and preventive strategies. Drugs Aging. 2012;29(5):359-376.

33. van der Velde N, Stricker BH, Pols HA, van der Cammen TJ. Risk of falls after withdrawal of fall-risk-increasing drugs: a prospective cohort study. Br J Clin Pharmacol. 2007;63(2):232-237.

34. Manning WJ. Asymptomatic aortic stenosis in the older people: a clinical review. JAMA. 2013;310(14):1490-1497.

35. Nkomo VT, Gardin JM, Skelton TN, Gottdiener JS, Scott CG, EnriquezSarano M. Burden of valvular heart diseases: a population-based study. Lancet. 2006;368(9540):1005-1011.

36. Fasano A, Plotnik M, Bove F, Berardelli A. The neurobiology of falls. Neurol Sci. 2012;33(6):1215-1223.

37. Ferrucci L, Guralnik JM, Cavazzini C, et al. The frailty syndrome: a critical issue in geriatric oncology. Crit Rev Oncol Hematol. 2003; 46(2):127-137.

38. Chen MA. Frailty and cardiovascular disease: potential role of gait speed in surgical risk stratification in older adults. J Geriatr Cardiol. 2015; 12(1):44-56
39. Tanimoto Y, Watanabe M, Sun W, et al. Sarcopenia and falls in community-dwelling older people subjects in Japan: defining sarcopenia according to criteria of the European Working Group on sarcopenia in older people. Arch Gerontol Geriatr. 2014;59(2):295-299.

40. Bien B, Wilmanska J, Janczak W, et al. Syncope and near-syncope as a multifactorial problem in geriatric inpatients: systemic hypotension is an underrated predictor for syncope exclusively. Adv Med Sci. 2011; 56(2):352-360.

41. Pothula VB, Chew F, Lesser TH, Sharma AK. Falls and vestibular impairment. Clin Otolaryngol Allied Sci. 2004;29(2):179-182.

42. Ungar A, Mussi C, Nicosia F, et al. The "syncope and dementia" study: a prospective, observational, multicenter study of elderly patients with dementia and episodes of "suspected" transient loss of consciousness. Aging Clin Exp Res. 2015;27(6):877-882.

43. Menant JC, Wong AK, Trollor JN, Close JC, Lord SR. Depressive symptoms and orthostatic hypotension are risk factors for unexplained falls in community-living older people. J Am Geriatr Soc. 2016;64(5): 1073-1078.

44. Chang VC, Do MT. Risk factors for falls among seniors: implications of gender. Am J Epidemiol. 2015;181(7):521-531.

45. Chen X, Mao G, Leng SX. Frailty syndrome: an overview. Clin Interv Aging. 2014;9:433-441
Clinical Interventions in Aging

\section{Publish your work in this journal}

Clinical Interventions in Aging is an international, peer-reviewed journal focusing on evidence-based reports on the value or lack thereof of treatments intended to prevent or delay the onset of maladaptive correlates of aging in human beings. This journal is indexed on PubMed Central, MedLine,

\section{Dovepress}

CAS, Scopus and the Elsevier Bibliographic databases. The manuscript management system is completely online and includes a very quick and fair peer-review system, which is all easy to use. Visit http://www.dovepress. com/testimonials.php to read real quotes from published authors. 